\title{
BMJ Open Role of hypertension in the association of overweight and obesity with diabetes among adults in Bangladesh: a population-based, cross-sectional nationally representative survey
}

Md Belal Hossain (D) ,,2 Jahidur Rahman Khan (D) ,, Rajat Das Gupta (D) 1,5

To cite: Hossain MB, Khan JR, Das Gupta R. Role of hypertension in the association of overweight and obesity with diabetes among adults in Bangladesh: a populationbased, cross-sectional nationally representative survey. BMJ Open 2021;11:e050493. doi:10.1136/ bmjopen-2021-050493

- Prepublication history and additional supplemental material for this paper are available online. To view these files, please visit the journal online (http://dx.doi.org/10.1136/ bmjopen-2021-050493)

Received 22 February 2021 Accepted 20 July 2021
Check for updates

(C) Author(s) (or their employer(s)) 2021. Re-use permitted under CC BY-NC. No commercial re-use. See rights and permissions. Published by BMJ.

For numbered affiliations see end of article.

Correspondence to

Md Belal Hossain;

bhossain@isrt.ac.bd

\section{ABSTRACT}

Aims Overweight and obesity (OWOB) is a modifiable risk factor for both hypertension and diabetes. However, the association between OWOB and diabetes among Bangladeshi adults and how hypertension may mediate this relationship are not well explored. This study aimed to examine (1) whether OWOB is independently associated with diabetes among Bangladeshi adults, (2) whether this association is mediated by hypertension, and (3) the effect modification by wealth status and place of residence in the relationships.

Research design and methods We used data of 9305 adults aged $\geq 18$ years from the most recent nationally representative cross-sectional study of Bangladesh Demographic and Health Survey 2017-2018. Design-based logistic regression was used to assess the association between OWOB and diabetes, and counterfactual framework-based weighting approach was used to evaluate the mediation effect of hypertension in the OWOB-diabetes relationship. We used stratified analyses for the effect modifications.

Results The prevalence of OWOB, diabetes and hypertension was $48.5 \%, 11.7 \%$ and $30.3 \%$, respectively. We observed a significant association between OWOB and diabetes and a mediating role of hypertension in the OWOB-diabetes association. The odds of diabetes was $51 \%$ higher among adults with OWOB than those without OWOB (adjusted OR: $1.51,95 \% \mathrm{Cl} 1.29$ to 1.77 ). We observed that $18.64 \%(95 \% \mathrm{Cl} 9.84 \%$ to $34.07 \%)$ of the total effect of OWOB on the higher odds of diabetes was mediated through hypertension, and the mediation effect was higher among adults from non-poor households and from both rural and urban areas.

Conclusions Adult OWOB status is independently associated with diabetes in Bangladesh, and hypertension mediates this association. Therefore, prevention policies should target adults with both OWOB and hypertension, particularly those from non-poor households and from both rural and urban areas, to reduce the growing burden of diabetes and its associated risk.

\section{INTRODUCTION}

Diabetes mellitus is a major global public health concern and is the leading cause
Strengths and limitations of this study

- This is the first study to investigate the mediating role of hypertension in the overweight and obesity (OWOB)-diabetes relationship among adults in Bangladesh.

- The sample size was large and representative of the population aged 18 years or older.

- All analyses were appropriately adjusted for survey cluster, strata and weights to account for the complex survey design.

- The study analysed cross-sectional data, which precludes the assessment of temporal relationships.

- More than one mediator variable, such as physical activity and diet, could mediate the OWOB-diabetes relationship.

of mortality and disability. ${ }^{12}$ The number of people with diabetes increased from 108 million in 1980 to 422 million in $2014 .^{3}$ Besides, diabetes is directly responsible for 1.6 million global deaths, while high blood glucose is responsible for another 2.2 million deaths. In Bangladesh, the prevalence of diabetes increased from $12 \%$ in 2011 to $14 \%$ in 2018 among men and from $11 \%$ in 2011 to $14 \%$ in 2018 among women aged 35 years or older. ${ }^{4}$ It is projected that more than 13.7 million people will have diabetes by $2045 .{ }^{5}$ Overweight and obesity (OWOB) is also a major public health concern in Bangladesh, leading to risk factors for many chronic noncommunicable diseases (NCDs). Due to the increased prevalence of OWOB and diabetes, this concern is termed as the '21st century epidemic'. ${ }^{6}$ The prevalence of OWOB among Bangladeshi adults increased from 23\% in 2011 to $35 \%$ in $2017-2018$. While diabetes mellitus relies on many factors, its association with OWOB is well established in the literature. ${ }^{7-9}$ A meta-analysis of prospective cohort 
studies reported three times higher risk of diabetes among adults with OWOB. ${ }^{10}$ Studies based in Bangladesh also reported a greater risk of diabetes among adults with OWOB. ${ }^{11-13}$ However, the underlying mechanism of this increased risk of diabetes in adults with OWOB has yet to be investigated in Bangladesh.

OWOB plays a significant role in screening and determining high-risk patients with diabetes. ${ }^{14}$ Since the prevalence of both OWOB and diabetes is strikingly increasing, Zimmet $e t a l^{15}$ termed the problem as 'diabesity' to exemplify the interdependence between these two major public health concerns. OWOB is also a modifiable risk factor for hypertension. Previous studies reported that OWOB and its associated metabolic abnormalities increase the risk of hypertension. ${ }^{16-19}$ Studies conducted in Bangladesh also found that OWOB is a risk factor for hypertension. ${ }^{20-22}$ Previous studies ${ }^{1122123}$ also pointed out wealth status and rural-urban discrepancies in the prevalence of OWOB, hypertension and diabetes. Since Bangladesh is going through an epidemiological and nutritional transition (ie, a high diet in total fat, cholesterol, sugar and other developed carbohydrates and little in polyunsaturated fatty acids and fibre) and experiencing rapid urbanisation, assessing the OWOB-diabetes relationship and the role of hypertension in this association would be critical to perform in this context.

The relationship between hypertension and diabetes is complicated, but both are associated with an elevated risk of cardiovascular diseases. Furthermore, many previous studies suggested that hypertension increases the risk of diabetes. In other words, hypertension is considered an independent risk factor for diabetes. ${ }^{24-28}$ For instance, a cohort study in the $\mathrm{UK},{ }^{29}$ which included 4.1 million adults, reported $52 \%-77 \%$ increased risk of diabetes with increasing blood pressure (BP). Notably, the frequent coexistence of diabetes and hypertension in the same subject is often considered a coincidence. However, many previous studies explained that patients with hypertension and diabetes share some common aspects of pathophysiology, particularly those related to OWOB and insulin resistance. ${ }^{2430} 31$

Hypertension is widely considered a confounding variable in the association between OWOB and diabetes. ${ }^{1432} 33$ However, hypertension could be a mediator or a collider in the OWOB-diabetes relationship. Consider the hypothetical examples in online supplemental figures $\mathrm{S} 1$ and S2, where we want to explore the total effect of OWOB on diabetes. In online supplemental figure S1, OWOB causes hypertension, and hypertension causes diabetes; therefore, hypertension is a mediator (or an intermediate variable) in the OWOB-diabetes relationship. In online supplemental figure S2, hypertension is the effect of both OWOB and diabetes; therefore, hypertension is a collider in the OWOB-diabetes relationship. Adjusting for a mediator leads to decompose the total effect into direct and indirect effects, ${ }^{34}$ while adjusting for a collider introduces bias. ${ }^{35}$ Therefore, adjusting for hypertension will bias the OWOB-diabetes relationship in both examples.
This study had three aims. First, we assessed the association between OWOB and diabetes among the adult population in Bangladesh using the most recent nationally representative data. Second, we evaluated the mediating role of hypertension in the OWOB-diabetes relationship. Third, we explored the effect modification by wealth status and place of residence in the OWOB-diabetes relationship in the total effect and the mediated effect through hypertension. To the best of our knowledge, this is the first ever study in Bangladesh to explore the mediation effect of hypertension in the OWOB and diabetes relationship and will guide future studies in a similar context.

\section{METHODS \\ Data preparation}

Data used in this study were extracted from the most recent nationally representative Bangladesh Demographic and Health Survey (BDHS) 2017-2018. A two-stage stratified cluster random sampling design was applied to collect the sample. In this survey, survey clusters (250 from urban stratum and 425 from rural stratum) were selected with a probability proportional to cluster size in the first stage. The BDHS 2017-2018 used a list of enumeration areas (ie, clusters) as a sampling frame, gathered from the 2011 Population and Housing Census of the People's Republic of Bangladesh. Each cluster consists of an average of about 120 households. In the second stage, a systematic sample of an average of 30 households per cluster was chosen. The BDHS 2017-2018 report provides a detailed methodology about survey sampling. ${ }^{4}$ For the present study, 13252 participants aged $\geq 18$ years selected for blood glucose measurements were considered. Individuals were excluded in this study owing to missing or implausible blood glucose measurement, pregnant women, not the usual resident of the selected households, missing values in body mass index (BMI), underweight (BMI $<18.5 \mathrm{~kg} /$ $\mathrm{m}^{2}$ ) and having missing values in covariates (see figure 1 for details). The final analytic sample consisted of 9305 unweighted (9136 weighted) adults aged 18 years or older with a BMI of $\geq 18.5 \mathrm{~kg} / \mathrm{m}^{2}$.

\section{Study variables \\ Outcome}

Diabetes status was used as the outcome variable for this study. Individuals were considered to have diabetes if they had a fasting blood glucose equivalent level of $\geq 7 \mathrm{mmol} / \mathrm{L}$ or reported taking prescribed medication to reduce high blood glucose or diabetes. ${ }^{4}$

Blood glucose was measured using the HemoCue 201 RT analyser. The sample collection technique was described in the BDHS 2017-2018 final report. ${ }^{4}$

\section{Exposure}

The exposure variable of interest was OWOB, created based on the Asian BMI cut-off. ${ }^{36}$ Individuals with a BMI of $\geq 23.0 \mathrm{~kg} / \mathrm{m}^{2}$ were categorised as overweight and obese. 


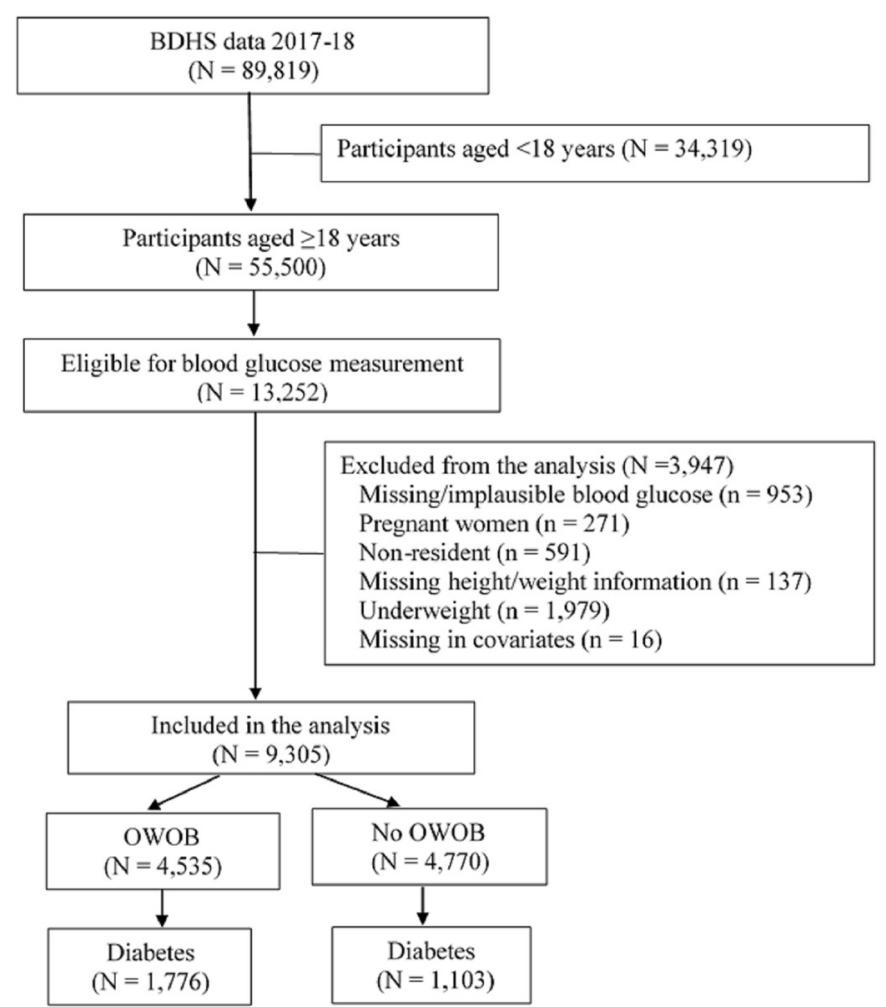

Figure 1 Flow chart showing the selection of adults aged 18 years or older for the present study from the 2017-2018 Bangladesh Demographic and Health Survey (BDHS) secondary data set. OWOB, overweight and obesity.

BMI was calculated as the ratio of weight in kilograms to the square of height in metres (ie, $\mathrm{kg} / \mathrm{m}^{2}$ ). Respondents' weight was obtained using lightweight electronic SECA 878 scales with a digital screen, while standing height was measured using measuring boards.

\section{Mediator}

Hypertension status was the mediator variable for this study. Individuals with an average systolic BP $\geq 140 \mathrm{~mm}$ $\mathrm{Hg}$, or diastolic $\mathrm{BP} \geq 90 \mathrm{~mm} \mathrm{Hg}$, or taking antihypertensives were categorised as hypertensive. ${ }^{437}$

BDHS used the LifeSource UA-767 Plus BP monitor, an automatic device, to measure BP. Trained health technicians measured three BP measurements at approximately $10 \mathrm{~min}$ intervals. The average of the second and third measurements was used to report BP values. Details can be found in the BDHS 2017-2018 report. ${ }^{4}$

\section{Effect modifiers}

In this study, household wealth status (poorest, poorer, middle, richer and richest) and place of residence (rural and urban) were the effect modifiers. We merged the poorest and poorer categories of wealth status variable into the poor class and other types into the non-poor class.

\section{Covariates}

Based on previous literature, ${ }^{11-13} \quad 20-22$ the following covariates were used to adjust for potential confounders: age $(<35,35-49,50-64$ and $\geq 65$ years), sex (male or
Common set of confounding variables: age, sex, marital status, education, working status, household wealth status, place of residence, and division of residence

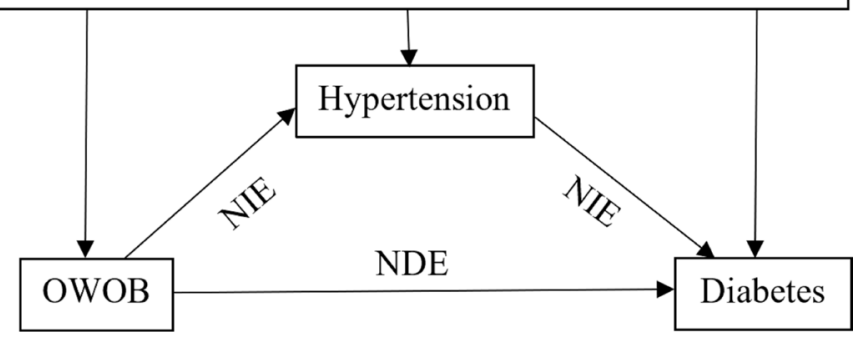

Figure 2 Diagram showing the hypothesised relationship of overweight and obesity (OWOB) with diabetes among the adult population in Bangladesh. The natural direct effect (NDE) of OWOB on diabetes is the primary effect of OWOB on diabetes (OWOB $\rightarrow$ diabetes), and the natural indirect effect (NIE) is mediated through hypertension (OWOB $\rightarrow$ hypertension $\rightarrow$ diabetes).

female), marital status (never married, currently married and others), education (no education, primary incomplete, primary or secondary incomplete, and secondary or higher), working status (yes or no), household wealth status, place of residence and division of residence (Barishal, Chattogram, Dhaka, Khulna, Mymensingh, Rajshahi, Rangpur and Sylhet).

\section{Statistical analysis}

We had only $16(0.17 \%)$ missing values in the covariates (figure 1). Therefore, the complete case analysis that justifies the missing completely at random assumption seems to be satisfied in this study. All analyses were appropriately adjusted for survey weights, clusters and strata to account for the complex survey design. Descriptive statistics were calculated to assess the distribution of variables. Design-based binary logistic regression ${ }^{38}$ was used to determine the crude and adjusted association of OWOB with diabetes.

A counterfactual framework-based weighting approach ${ }^{3940}$ was used to explore the effect of OWOB on diabetes into (1) natural direct effect (NDE), that is, the direct effect of OWOB on diabetes not mediated through hypertension; and (2) natural indirect effect (NIE), that is, the effect of OWOB on diabetes mediated through hypertension (figure 2). Analyses were adjusted for the confounding variables age, sex, marital status, education, working status, household wealth status, place of residence and division of residence. We multiplied the mediation weights and the survey weights to get the survey-based estimates. ${ }^{40-42}$ The odds ratio (OR) for the total effect was the product of the OR for the NDE and the NIE. We considered 250 bootstrap replicates to obtain the $95 \%$ CI of the total effect, NDE, NIE and proportion mediated, where proportion mediated was calculated as dividing NIE in logarithm scale by total effect in logarithm scale. ${ }^{4042}$

We used the above-mentioned counterfactual framework-based weighting approach to explore the effect modification by household wealth status and place 
of residence. We implemented the above-described methods to calculate the total effect, NDE, NIE and proportion mediated separately for the poor, non-poor, rural and urban groups.

As a sensitivity analysis, we categorised the hypertension variable using the American College of Cardiology (ACC) and the American Heart Association (AHA) guidelines. ${ }^{43}$ Individuals with average systolic $\mathrm{BP} \geq 130 \mathrm{~mm} \mathrm{Hg}$, or diastolic $\mathrm{BP} \geq 80 \mathrm{~mm} \mathrm{Hg}$, or taking antihypertensives were categorised as hypertensive. Then we applied the same technique as described above to explore the effect of OWOB on diabetes into NDE and NIE. All analyses were conducted using the statistical software R V.4.0.3. ${ }^{44}$

\section{Patient and public involvement}

Patients and other members of the public were not involved in the study.

\section{RESULTS}

\section{Study sample characteristics}

Descriptive statistics of the variables stratified by OWOB are presented in table 1 . Among the selected individuals, $48.5 \%$ were overweight and obese and $11.7 \%$ had diabetes. The prevalence of hypertension was $30.3 \%$, while it was $60.0 \%$ based on the ACC-AHA guideline. Approximately $43.1 \%$ of the study participants were $<35$ years old, $56.8 \%$ were female, $82.4 \%$ were currently married, $23.8 \%$ had no education, $19.3 \%$ had completed secondary or higher education, $62.6 \%$ were currently working, $23.4 \%$ were from Dhaka division, $71.7 \%$ were from rural areas, and $35.6 \%$ were from poor households. The prevalence of diabetes was comparatively higher among adults with OWOB ( $15.1 \%$ vs $8.4 \%)$. A higher proportion of individuals with $\mathrm{OWOB}$ were hypertensive, female, completed secondary or higher education, from urban areas and from non-poor households.

\section{Association between OWOB and diabetes}

Table 2 shows the crude and adjusted association between OWOB and diabetes. In the crude analysis, the odds of diabetes was $94 \%$ higher among adults with OWOB compared with those without OWOB (OR: 1.94, 95\% CI 1.67 to 2.24). We also observed a significant association between OWOB and diabetes after adjusting the model for potential confounders. We found that the adjusted odds of diabetes was $51 \%$ higher among adults with OWOB than those without OWOB (adjusted OR (AOR): $1.51,95 \%$ CI 1.29 to 1.77 ).

\section{Role of hypertension in the OWOB-diabetes association}

In estimating the mediating effect, we first modelled the relationship of hypertension (the mediator) with OWOB exposure and diabetes outcome. We found that OWOB was significantly associated with higher odds of hypertension (AOR: 2.28, 95\% CI 2.03 to 2.57). The mediator was also strongly associated with diabetes (AOR: 1.69, 95\% CI 1.45 to 1.97$)$.
Finally, we implemented the counterfactual frameworkbased weighting approach to estimate the mediating effect. Table 3 shows the NDE of OWOB on diabetes and the NIE of OWOB on diabetes mediated through hypertension. A $50 \%$ higher odds of diabetes among adults with OWOB was deconstructed into a direct OR of $1.39(95 \%$ CI 1.18 to 1.65$)$ and a hypertension-mediated indirect OR of 1.08 (95\% CI 1.05 to 1.12). Approximately $18.64 \%$ (95\% CI $9.84 \%$ to $34.07 \%$ ) of the total effect of OWOB on the higher odds of diabetes was mediated through hypertension.

\section{Effect modification by wealth status and place of residence}

The results of effect modification by wealth status and place of residence are also shown in table 3 . The odds of diabetes was $70 \%$ higher among adults with OWOB compared with those without OWOB in poor households (AOR: $1.70,95 \%$ CI 1.25 to 2.37), 59\% higher in nonpoor households (AOR: 1.59, 95\% CI 1.33 to 1.96 ), $55 \%$ higher in rural areas (AOR: 1.55, 95\% CI 1.27 to 1.89 ) and $42 \%$ higher in urban areas (AOR: 1.42, 95\% CI 1.10 to 1.89). We observed that the total effect of OWOB on the higher odds of diabetes mediated through hypertension was comparatively higher among adults from nonpoor households than poor households $(20.0 \%$ vs $6.1 \%)$ and urban areas than rural areas $(26.8 \%$ vs $16.1 \%)$.

\section{Sensitivity analyses}

Online supplemental table 1 shows the results of the sensitivity analysis of the NDE of OWOB on diabetes and the NIE of OWOB on diabetes mediated through hypertension, where hypertension was defined based on the ACC-AHA guideline. Similar to the primary analysis, we observed a statistically significant direct and hypertensionmediated indirect effect of OWOB on diabetes. The results were also consistent for the stratified analyses.

\section{DISCUSSION}

Using the most recent national survey data, we found that OWOB is an independent risk factor for diabetes in Bangladesh. Adults with OWOB were $51 \%$ more likely to have diabetes than their counterparts. We found a similar strength of association using the counterfactual framework-based weighting approach. Besides, hypertension substantially mediated the OWOB-diabetes relationship. There was a comparatively higher mediated effect of hypertension in the OWOB-diabetes relationship among adults from non-poor households than from poor households and from urban areas than rural areas. A sensitivity analysis using a different definition of hypertension revealed consistent results. These findings contribute to a better understanding of the relationship between OWOB and diabetes and the mediation effects of hypertension in the OWOB-diabetes association needed to develop appropriate preventive strategies.

In line with earlier studies, ${ }^{1-13}$ adults with OWOB were more likely to develop diabetes. The potential reason 
Table 1 Characteristics of the study sample $(\mathrm{N}=9305)$ by $\mathrm{OWOB}^{*}$ among adults aged 18 years or older in the Bangladesh Demographic and Health Survey 2017-2018.

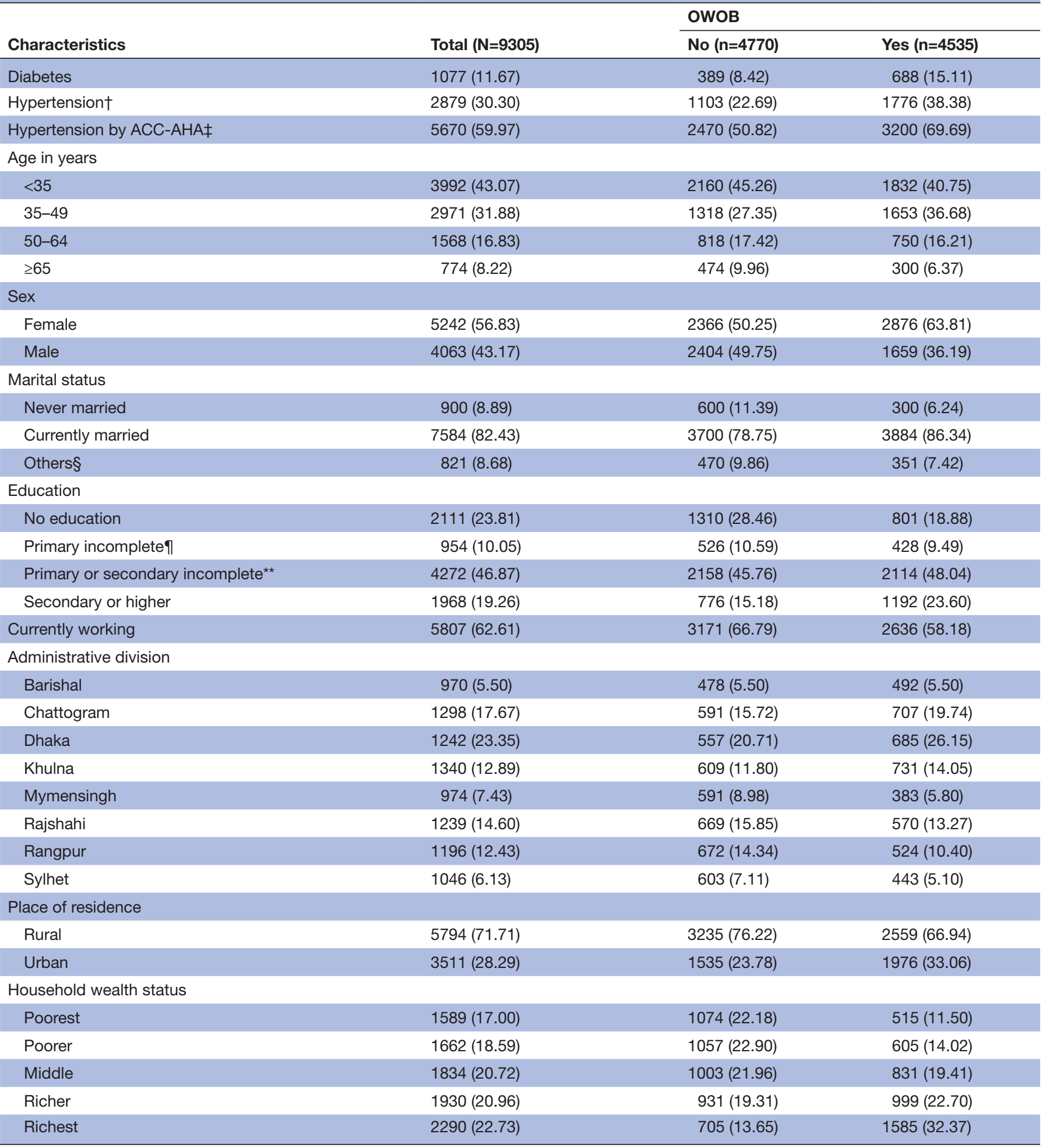

${ }^{*}$ Except where indicated otherwise, values are number (\%), where the number comes from the sample and \% comes from the survey design that accounts for survey features such as strata, cluster and survey weights.

†Individuals with average systolic blood pressure $\geq 140 \mathrm{~mm} \mathrm{Hg}$, or diastolic blood pressure $\geq 90 \mathrm{~mm} \mathrm{Hg}$, or taking antihypertensives were categorised as hypertensive.

†Individuals with average systolic blood pressure $\geq 130 \mathrm{~mm} \mathrm{Hg}$, or diastolic blood pressure $\geq 80 \mathrm{~mm} \mathrm{Hg}$, or taking antihypertensives were categorised as hypertensive.

§Divorced, separated or widowed.

IPrimary incomplete means completing grades 1-4.

**Primary or secondary incomplete means completing grades 5-9.

ACC, American College of Cardiology; AHA, American Heart Association; OWOB, overweight and obesity. 
Table 2 Unadjusted and adjusted associations between OWOB and diabetes among adults aged 18 years or older in Bangladesh.

\begin{tabular}{|c|c|c|}
\hline \multirow{2}{*}{$\begin{array}{l}\text { Exposure of } \\
\text { interest }\end{array}$} & Unadjusted & Adjusted* \\
\hline & COR (95\% CI) & AOR $(95 \% \mathrm{Cl})$ \\
\hline \multicolumn{3}{|l|}{ OWOB } \\
\hline No & Ref & Ref \\
\hline Yes & 1.94 (1.67 to 2.24$)$ & 1.51 (1.29 to 1.77$)$ \\
\hline
\end{tabular}

*Adjusted for age, sex, marital status, education, working status, division of residence, place of residence and household wealth status.

AOR, adjusted OR; COR, crude/unadjusted OR; OWOB, overweight and obesity; Ref, reference.

for this result could be insulin resistance; however, the full pathways of this association have not been elucidated. The quantity of non-esterified fatty acids, glycerol, hormones, cytokines, proinflammatory substances and other substances is related to insulin resistance development among individuals with OWOB, and insulin resistance leads to the development of diabetes. ${ }^{9}$ Also, in obesity, there is a dysfunction of $\beta$ cell, and this leads to diabetes. ${ }^{9}$ Therefore, preventing the burden of OWOB would lead to prevention of diabetes. The higher prevalence of OWOB is attributed to poor dietary habits, higher physical inactivity, higher sedentary behaviours and other lifestyle-related factors. Loss of weight can reduce the risk of diabetes, as loss of weight may enhance the action of insulin and the concentration of fasting glucose. ${ }^{45}$ Therefore, public health programmes for changing diets and lifestyles can prevent adults from developing OWOB and reduce the burden of diabetes.

We observed that the association between OWOB and diabetes among Bangladeshi adults was substantially mediated by hypertension. More than $18 \%$ of the total effect of OWOB on the higher odds of diabetes was mediated through hypertension. It is, therefore, essential to understanding the underlying mechanism of being a mediator. The mechanism by which OWOB is linked to hypertension may include activation of the sympathetic nervous system and the renin-angiotensin-aldosterone system, endothelial dysfunction and renal functional abnormalities. ${ }^{46}$ The pathophysiology of linking hypertension with the risk of diabetes is still not clear; however, there are several hypotheses. ${ }^{28}$ Microvascular dysfunction induced by hypertension leads to the development of diabetes. Also, endothelial dysfunction is associated with insulin resistance, which leads to diabetes development. Further, inflammatory markers are associated with both hypertension and diabetes.

In earlier research in Bangladesh, ${ }^{11} 1347$ hypertension was considered a predictor of diabetes, and it was not fully clarified whether hypertension acts as a confounder, mediator or collider. However, hypertension should be considered a mediator or a collider but not a confounder in the OWOB-diabetes relationship (see online supplemental figures 1 and 2). Since adjusting for a mediator in the regression analysis decomposes the total effect into direct and indirect effects, ${ }^{34}$ we should not adjust for hypertension in the OWOB and diabetes association analysis if our goal is to get the total effect. Moreover, since adjusting for a collider introduces bias, ${ }^{35}$ we should not adjust for hypertension in the OWOB and diabetes association analysis. Thus, regardless of considering hypertension as a mediator or a collider, we should not consider it an adjustment variable in the regression analysis of the total effect of the OWOB-diabetes association. Future diabetes research should take care of this factor during their analysis. In the present study, we gave a concrete explanation of the mediating role of hypertension in the OWOB-diabetes relationship. Future studies could conduct the mediation analysis in a similar context. For example, future studies could explore the mediating role of diet in the OWOB-diabetes relationship.

Mediation analysis also exhibits wealth group and ruralurban differences in the role of hypertension as a mediator in the OWOB-diabetes association, with the highest contribution in non-poor and urban adults. The higher mediation effect of hypertension among adults from nonpoor households might be due to the risk of hypertension among those adults. Therefore, we recommend targeting adults with both OWOB and hypertension, particularly those from non-poor households from both rural and

Table 3 Total, direct and hypertension-mediated ${ }^{*}$ effect of OWOB $\dagger$ on diabetes in Bangladesh.

\begin{tabular}{|c|c|c|c|c|}
\hline & Total effect & NDE & NIE & \\
\hline Group & AOR $(95 \% \mathrm{Cl})$ & AOR $(95 \% \mathrm{Cl})$ & AOR $(95 \% \mathrm{Cl})$ & Mediation effect, $\%$ \\
\hline Overall & 1.50 (1.27 to 1.76$)$ & 1.39 (1.18 to 1.65$)$ & 1.08 (1.05 to 1.12$)$ & 18.64 \\
\hline Non-poor & 1.59 (1.33 to 1.96$)$ & 1.45 (1.20 to 1.83$)$ & 1.10 (1.06 to 1.14$)$ & 19.97 \\
\hline Rural & 1.55 (1.27 to 1.89$)$ & 1.44 (1.17 to 1.80$)$ & 1.07 (1.03 to 1.12 ) & 16.11 \\
\hline
\end{tabular}

*Individuals with average systolic blood pressure $\geq 140 \mathrm{~mm} \mathrm{Hg}$, or diastolic blood pressure $\geq 90 \mathrm{~mm}$ Hg, or taking antihypertensives were categorised as hypertensive.

†The reference was individuals without OWOB.

AOR, adjusted OR; NDE, natural direct effect; NIE, natural indirect effect; OWOB, overweight and obesity. 
urban areas, to reduce the growing burden of diabetes in Bangladesh. Since OWOB is a modifiable risk factor for hypertension and diabetes, efforts should be taken to reduce the prevalence of OWOB. Notably, OWOB is interconnected with many other lifestyle factors, such as physical activity, diet, smoking and alcohol consumption. Therefore, public health policies could focus on proper behavioural change communication, initiating and properly maintaining innovative technologies, cost-effective strategies (eg, increasing physical activity, maintaining a good diet, cessation of smoking, screening) and lifestyle interventions. These techniques are proven useful tools for preventing overall NCD burden in low-income and middle-income country settings. ${ }^{48}$

The present study has several strengths. To the best of our knowledge, this study is the first to assess the association between OWOB and diabetes in Bangladesh and estimate the role of hypertension in the OWOB-diabetes relationship among adults using mediation analysis. Overall, adults with an overnourished profile exhibited a higher risk of suffering from diabetes, and hypertension acts as a mediator in the relationship between OWOB and diabetes. Moreover, this study has used robust statistical techniques to estimate a reliable association and reduce bias, such as design-based binary logistic regression and counterfactual frameworkbased weighting approach.

This study also has some limitations that need to be acknowledged. First, this study's cross-sectional nature prevents us from evaluating the temporal relationship between the exposure, mediator and outcome. Second, generalisations based on our findings may be limited due to the complex effects of OWOB on diabetes in adults and having unmeasured confounders, such as dietary and physical activity behaviour, genetics and environmental influences, and regional variability. Future studies could use the data from the WHO STEPwise Approach to NCD Risk Factor Surveillance (STEPS) survey in Bangladesh which collected information on many covariates that are associated with NCDs. ${ }^{49} 50$ However, the WHO STEPS survey results could not be generalisable to all adults since the study was restricted to adults aged 18-69 years old only. Third, we did not use the standardised methods using fasting venous plasma glucose, repeat measurements or haemoglobin A1c to diagnose diabetes. Therefore, our estimates are likely to be underestimated since our random glucose definition is conservative (more specific but less sensitive).$^{51}$ Finally, the OWOB-diabetes relationship is likely to be mediated by more than one mediator variable, such as physical activity and diet. Future studies could explore the mediation role of these factors in the OWOB-diabetes relationship.

\section{CONCLUSION}

We found that OWOB is an independent risk factor for diabetes among the adult population in Bangladesh. We observed that adults with $\mathrm{OWOB}$ were more than twice more likely to be diabetic compared with those without
OWOB. We also observed a significant mediating role of hypertension in the OWOB-diabetes association. Approximately $18.64 \%$ of the total effect of OWOB was mediated through hypertension, and the mediation effect was pronounced among those adults from non-poor households and both rural and urban areas. Thus, the targeting of adults with OWOB and hypertension, particularly those from non-poor households from both the rural and urban areas, could be an effective public health strategy to reduce the burden of diabetes and improve the quality of life among the adult population in Bangladesh.

\section{Author affiliations}

${ }^{1}$ BRAC James P Grant School of Public Health, BRAC University, Dhaka, Bangladesh ${ }^{2}$ School of Population and Public Health, University of British Columbia, Vancouver, British Columbia, Canada

${ }^{3}$ Biomedical Research Foundation, Dhaka, Bangladesh

${ }^{4}$ Health Research Institute, Faculty of Health, University of Canberra, Bruce, Canberra, Australia

${ }^{5}$ Department of Epidemiology and Biostatistics, Arnold School of Public Health, University of South Carolina, Columbia, South Carolina, USA

Acknowledgements We would like to thank MEASURE DHS for granting access to the BDHS data sets. We would also like to thank the editor and referees for critical readings and helpful comments.

Contributors MBH takes responsibility for the integrity of the data and the accuracy of the data analysis. MBH conceptualised the analysis plan and performed the statistical analysis. MBH and JRK interpreted the results and drafted the paper together. RDG revised it critically for important intellectual content and contributed to editing the first draft of the manuscript. All authors have reviewed and approved the final manuscript.

Funding The authors have not declared a specific grant for this research from any funding agency in public, commercial or not-for-profit sectors.

Competing interests None declared.

Patient consent for publication Not required.

Ethics approval The 2017-2018 BDHS is a secondary and publicly accessible data set. Therefore, this present study was exempted from ethics approval. However, the 2017-2018 BDHS was reviewed and approved by the Ministry of Health and Family Welfare. The survey was implemented under the authority of the National Institute of Population Research and Training (NIPORT), Medical Education and Family Welfare Division, Ministry of Health and Family Welfare.

Provenance and peer review Not commissioned; externally peer reviewed.

Data availability statement Data are available in a public, open access repository. BDHS data are publicly accessible upon request from the DHS website at http:// dhsprogram.com/data/available-datasets.cfm.

Supplemental material This content has been supplied by the author(s). It has not been vetted by BMJ Publishing Group Limited (BMJ) and may not have been peer-reviewed. Any opinions or recommendations discussed are solely those of the author(s) and are not endorsed by BMJ. BMJ disclaims all liability and responsibility arising from any reliance placed on the content. Where the content includes any translated material, BMJ does not warrant the accuracy and reliability of the translations (including but not limited to local regulations, clinical guidelines, terminology, drug names and drug dosages), and is not responsible for any error and/or omissions arising from translation and adaptation or otherwise.

Open access This is an open access article distributed in accordance with the Creative Commons Attribution Non Commercial (CC BY-NC 4.0) license, which permits others to distribute, remix, adapt, build upon this work non-commercially, and license their derivative works on different terms, provided the original work is properly cited, appropriate credit is given, any changes made indicated, and the use is non-commercial. See: http://creativecommons.org/licenses/by-nc/4.0/.

ORCID iDs

Md Belal Hossain http://orcid.org/0000-0003-4603-863X

Jahidur Rahman Khan http://orcid.org/0000-0002-3029-6303

Rajat Das Gupta http://orcid.org/0000-0002-7680-676X 


\section{REFERENCES}

1 Lozano R, Naghavi M, Foreman K, et al. Global and regional mortality from 235 causes of death for 20 age groups in 1990 and 2010: a systematic analysis for the global burden of disease study 2010. Lancet 2012;380:2095-128.

2 Murray CJL, Vos T, Lozano R, et al. Disability-adjusted life years (DALYs) for 291 diseases and injuries in 21 regions, 1990-2010: a systematic analysis for the global burden of disease study 2010. Lancet 2012;380:2197-223.

3 WHO. Diabetes, 2020. Available: https://www.who.int/news-room/ fact-sheets/detail/diabetes\#: :text=The number of people with,in premature mortality from diabetes [Accessed 31 Dec 2020].

4 National Institute of Population Research and Training (NIPORT), ICF. Bangladesh demographic and health survey 2017-18. Dhaka Bangladesh, and Rockville, Maryland, USA, 2020.

5 Cho NH, Shaw JE, Karuranga S, et al. IDF diabetes atlas: global estimates of diabetes prevalence for 2017 and projections for 2045. Diabetes Res Clin Pract 2018;138:271-81.

6 Golay A, Ybarra J. Link between obesity and type 2 diabetes. Best Pract Res Clin Endocrinol Metab 2005;19:649-63.

7 Nguyen NT, Nguyen X-MT, Lane J, et al. Relationship between obesity and diabetes in a US adult population: findings from the National health and nutrition examination survey, 1999-2006. Obes Surg 2011;21:351-5.

8 Pi-Sunyer X. The medical risks of obesity. Postgrad Med 2009;121:21-33.

9 Al-Goblan AS, Al-Alfi MA, Khan MZ. Mechanism linking diabetes mellitus and obesity. Diabetes Metab Syndr Obes 2014;7:587.

10 Abdullah A, Peeters A, de Courten M, et al. The magnitude of association between overweight and obesity and the risk of diabetes: a meta-analysis of prospective cohort studies. Diabetes Res Clin Pract 2010;89:309-19.

11 Akter S, Rahman MM, Abe SK, et al. Prevalence of diabetes and prediabetes and their risk factors among Bangladeshi adults: a nationwide survey. Bull World Health Organ 2014;92:204-13.

12 Alam DS, Talukder SH, Chowdhury MAH, et al. Overweight and abdominal obesity as determinants of undiagnosed diabetes and pre-diabetes in Bangladesh. BMC Obes 2016;3:19.

13 Talukder A, Hossain MZ. Prevalence of diabetes mellitus and its associated factors in Bangladesh: application of two-level logistic regression model. Sci Rep 2020;10:1-7.

14 Wang S, Ma W, Yuan Z, et al. Association between obesity indices and type 2 diabetes mellitus among middle-aged and elderly people in Jinan, China: a cross-sectional study. BMJ Open 2016;6:e012742.

15 Zimmet P, Alberti KG, Shaw J. Global and societal implications of the diabetes epidemic. Nature 2001;414:782-7.

16 Hall JE, do Carmo JM, da Silva AA, et al. Obesity-induced hypertension: interaction of neurohumoral and renal mechanisms. Circ Res 2015;116:991-1006.

17 Redon J. Hypertension in obesity. Nutr Metab Cardiovasc Dis 2001;11:344-53.

18 Davy KP, Hall JE. Obesity and hypertension: two epidemics or one? Am J Physiol Regul Integr Comp Physiol 2004;286:R803-13.

19 Stamler R, Stamler J, Riedlinger WF, et al. Weight and blood pressure. findings in hypertension screening of 1 million Americans. JAMA 1978;240:1607-10.

20 Hossain FB, Adhikary G, Chowdhury AB, et al. Association between body mass index (BMI) and hypertension in South Asian population: evidence from nationally-representative surveys. Clin Hypertens 2019;25:28.

21 Kibria GMA, Swasey K, Hasan MZ, et al. Determinants of hypertension among adults in Bangladesh as per the joint National Committee 7 and 2017 American College of Cardiology/American hypertension association hypertension guidelines. J Am Soc Hypertens 2018;12:e45-55.

22 Tesfaye F, Nawi NG, Van Minh H, et al. Association between body mass index and blood pressure across three populations in Africa and Asia. J Hum Hypertens 2007;21:28-37.

23 Biswas T, Garnett SP, Pervin S, et al. The prevalence of underweight, overweight and obesity in Bangladeshi adults: data from a national survey. PLoS One 2017;12:e0177395.

24 Petrie JR, Guzik TJ, Touyz RM. Diabetes, hypertension, and cardiovascular disease: clinical insights and vascular mechanisms. Can J Cardiol 2018;34:575-84.

25 de Boer IH, Bangalore S, Benetos A, et al. Diabetes and hypertension: a position statement by the American diabetes association. Diabetes Care 2017;40:1273-84.
26 Adamsson Eryd S, Gudbjörnsdottir S, Manhem K, et al. Blood pressure and complications in individuals with type 2 diabetes and no previous cardiovascular disease: national population based cohort study. BMJ 2016;354:i4070.

27 Lewington S, Clarke R, Qizilbash N, et al. Age-specific relevance of usual blood pressure to vascular mortality: a meta-analysis of individual data for one million adults in 61 prospective studies. Lancet 2002;360:1903-13

28 Kim M-J, Lim N-K, Choi S-J, et al. Hypertension is an independent risk factor for type 2 diabetes: the Korean genome and epidemiology study. Hypertens Res 2015;38:783-9.

29 Emdin CA, Anderson SG, Woodward M, et al. Usual blood pressure and risk of new-onset diabetes: evidence from 4.1 million adults and a meta-analysis of prospective studies. J Am Coll Cardiol 2015:66:1552-62.

30 Lago RM, Singh PP, Nesto RW. Diabetes and hypertension. Nat Clin Pract Endocrinol Metab 2007;3:667.

31 Mendis S, O'Brien E, Seedat YK, et al. Hypertension and diabetes: entry points for prevention and control of the global cardiovascular epidemic. Int J Hypertens 2013;2013:1-3.

32 Mokdad AH, Ford ES, Bowman BA, et al. Prevalence of obesity, diabetes, and obesity-related health risk factors, 2001. JAMA 2003;289:76-9.

33 Sonmez A, Haymana C, Bayram F, et al. Turkish nationwide survEy of glycemic and other metabolic parameters of patients with diabetes mellitus (TEMD study). Diabetes Res Clin Pract 2018;146:138-47.

34 Richiardi L, Bellocco R, Zugna D. Mediation analysis in epidemiology: methods, interpretation and bias. Int J Epidemiol 2013;42:1511-9.

35 Cole SR, Platt RW, Schisterman EF, et al. Illustrating bias due to conditioning on a collider. Int J Epidemiol 2010;39:417-20.

36 WHO Expert Consultation. Appropriate body-mass index for Asian populations and its implications for policy and intervention strategies. Lancet 2004;363:157-63.

37 World Health Organization, International Society of Hypertension Writing Group. World Health Organization (WHO)/International Society of Hypertension (ISH) statement on management of hypertension. J Hypertens 2003;2003:1983-92.

38 Lumley T, Scott A. Fitting regression models to survey data. Stat Sci 2017;32:265-78.

39 Pearl J. Causality: models, Reasoning, and inference. 2nd ed. Los Angeles: Cambridge university press, 2013

40 Lange T, Vansteelandt S, Bekaert M. A simple unified approach for estimating natural direct and indirect effects. Am J Epidemiol 2012;176:190-5.

41 Austin PC, Jembere N, Chiu M. Propensity score matching and complex surveys. Stat Methods Med Res 2018;27:1240-57.

42 Rochon J, du Bois A, Lange T. Mediation analysis of the relationship between institutional research activity and patient survival. BMC Med Res Methodol 2014;14:9.

43 Whelton PK, Carey RM, Aronow WS, et al. 2017 ACC/AHA/AAPA ABC/ACPM/AGS/APhA/ASH/ASPC/NMA/PCNA guideline for the prevention, detection, evaluation, and management of high blood pressure in adults: a report of the American College of Cardiology/ American heart association Task force on clinical practice guidelines. Hypertension 2018;71:E13-115.

$44 \mathrm{R}$ Core Team. The R project for statistical computing, 2020. Available: https://www.r-project.org/ [Accessed 8 Jan 2021]

45 Rogers JZ, Still CD. Obesity and type 2 diabetes. Tampa, 2009.

46 Rahmouni K, Correia MLG, Haynes WG, et al. Obesity-Associated hypertension: new insights into mechanisms. Hypertension 2005;45:9-14.

47 Hussain A, Vaaler S, Sayeed MA, et al. Type 2 diabetes and impaired fasting blood glucose in rural Bangladesh: a population-based study. Eur J Public Health 2007;17:291-6.

48 Islam SMS, Purnat TD, Phuong NTA, et al. Non-Communicable diseases (NCDs) in developing countries: a symposium report. Global Health 2014;10:81.

49 WHO. National STEPS survey for non-communicable diseases risk factors in Bangladesh 2018, 2020. Available: https://apps.who.int/ iris/handle/10665/332886 [Accessed 6 Jul 2021]

50 Riaz BK, Islam MZ, Islam ANMS, et al. Risk factors for noncommunicable diseases in Bangladesh: findings of the populationbased cross-sectional national survey 2018. BMJ Open 2020;10:e041334.

51 Claypool KT, Chung M-K, Deonarine A, et al. Characteristics of undiagnosed diabetes in men and women under the age of 50 years in the Indian subcontinent: the National Family Health Survey (NFHS 4)/Demographic Health Survey 2015-2016. BMJ Open Diabetes Res Care 2020;8:e000965. 